\title{
GENDER STEREOTYPES AND STEAM EDUCATION
}

\author{
SORINA MIHAELA BĂLAN*, CAMELIA STANCIU \\ Dimitrie Cantemir University, Târgu Mureș, Romania \\ Corresponding author email: bsorinamihaela@yahoo.com
}

\begin{abstract}
Then we talk about gender stereotypes in Steam education we can find in the societal mentality: "Girls don't find STEAM interesting" „Boys are more capable for STEAM", „Boys are oriented to achievements, girls to feelings and society". This paper presents the results of sensitive gender workshops, to provide illustrations of stereotypes as an input for the creation of value-added content with gender awareness and continue sensitizing teachers about gender stereotypes in approach to learning that uses Science, Technology, Engineering, the Arts and Mathematics in education.
\end{abstract}

Key words: Gender Stereotips, STEAM Education, STEM Education, Sex Segregation.

\section{INTRODUCTION}

In a world with greater population, global interconnection, technological advancement, and large-scale problems than ever before in human history, complex problems require sophisticated problem solving skills and innovative, complicated solutions.[1]

Perignat \& Katz-Buonin examined 44 published articles (empirical, descriptive, and pedagogical frameworks) on the topic of STEAM education from 2007 to 2018 and also differentiate in methods for merging STEAM disciplines, described in one of five ways: transdisciplinary, interdisciplinary, multi-disciplinary, cross-disciplinary, and arts-integration.[2]

\section{MATERIAL AND METHODS}

\section{What meens STEAM Education?}

Tam, Yuk-fung Chan, \& Long-hin Lai propose to get optimal impact of STEM education and truly prepare students for the real world problems, a different approach of teaching where students can offer and argue with evidence, hear different points of view and discern which seem reasonable, interact and communicate newly created ideas is important [3]

Considering the current climate change, health disparities, food shortage, and others can say that STEM disciplines have become of greater importance than ever." [4]

Sex segregation seems to vary cross-nationally by socioeconomic development and gender equity; we may find similar variation on these indicators within this rapidly industrializing Southeast Asian nation. [5]

Students' beliefs, teachers' recommendation and the system of tracking are all important factors in choosing an in-demand science career. [6]

While education levels of women have increased dramatically relative to men, women 
are still greatly underrepresented in Science, Technology, Engineering, and Mathematics (STEM) college programmer.[7]

\section{The stereotyped situations in STEAM education}

Two workshops were performed in the field of STEAM Education. In first workshops participated 9 students (just girls) from the psychology faculty attended the second year, and in workshop 2: 16 students, including 14 girls and 2 boys, students from "Dimitrie Cantemir" University were studying Psychology, Law, and School-teaching. Workshop facilitators were provided with a guide to identify common and unconscious in stereotypes of gender in the most homogenous way as possible. The aims of sensitive gender workshops was: to provide a framework for input to encourage local content workshops; to provide illustrations of stereotypes as an input for the creation of value-added content with gender awareness; to continue sensitizing teachers about gender stereotypes in STEAM education.

\subsection{Breaking the ice activity}

The first activity comprised of the distribution of paper slips that contained statements on gender issues. The strips had been cut in half in various ways so that each piece could be only matched with its original mate.

In the second activity, participants had to find the matching half to the piece of paper they were holding. When they found the right match, they formed pairs with the person who had the matching piece. Each person in the pair then interviewed the other and after they discuss the sentence.

In the third activity, each person in each pair introduced his or her partner to the group after they had introduced themselves and been talking about their interests, etc Once they had introduced themselves, they had to say what phrase they had been touched by and what they thought of it, if they thought it was true, why?

Even though most of students agreed that it was not something of "nature" but of learned skills, it is worthy to remark that there is still a high percentage of students that consider that women cannot work as hard as men.

All participants presented and expressed their opinion freely. In the first group the opinions were homogeneous, they generally had the same opinions. In the second group, opinions were much more divergent and each tried to reasonably support his opinion.

Some of the initial views of the students are synthesized below:

- G. began the debate stating that "There are 60,000 anatomic differences between the body of the woman and the man: the most beautiful profession / profession is to form life through educated sittings" (C.G., Psychology, 1st year)

- T. S. say: "There is inequality between women and men. Unfortunately for the same thing in certain jobs even if women and men do the same job, men are better paid than women. I do not think men are always right"

- M.A. believes that "Women have the same rights as men" (MA-, future teacher) N.G. disagrees, says she was credited with a Muslim family, and women are honored, but she says, "I do not think we can be $100 \%$ equal. Men have certain qualities and women do not have them the other way around. We are different from each other "(N.G.-Muslimorigin third year psychology student, DPPD l)

- "In our society, geared towards democracy and equality, I believe that women use gender inequalities as a pretext to motivate their own failures. A woman who wants her education will be educated. A woman who wants to advance in her career will do everything in her power to get this done. If he wants a family and obligations; is involved, will establish a family, and if not, will not do so. Although stereotypes exist, they can't and must not stand in the way of a person's goals “(B.D.M.)

- P.S.A. says that "gender inequality exists today. Women are in some devalued areas, everything being in favor of men. They are not excluded, but only marginalized. Just as in rural areas, women are inferior to men because they are domestic, they have to deal with home and children in general, some of whom are denied the right to work. I think that even small children are involuntarily imposing certain stereotypes: women = secretary; 
man $=$ army; women = sensitivity, emotion; man $=$ force, protective. Each of these qualities, whether male or female, must be appreciated by each of the two parties, to complement each other." (DPPD student, first level, 3rd year of psychology)

- G.A.M., the mother of two boys says boys are disadvantaged, they are apostrophe more often than girls, sometimes even beaten by teenagers and girls are forgiven, even if they are wrong, they also conclude that "stores have more products for women than for men, what I do not feel right, I have two boys, go abroad to shop. For supplies and clothes, because we do not find in our country, women are more accented. I believe that society is first guilty of inducing this state of being unequal. Men love the real part (math-real), women's human side (the Romanian-human language). So they say. I say that it depends very much on how the little child is educated, it is about temperament, personality, character, no matter the genre. "

"Inequality is present and there will be a long time," adds a participant, and B.V. says "it is a controversial topic and I think most have a misconception about the role played by woman and man in society".

\subsection{Riddle activity}

The aim of activity has to get participants to re-think their first assumptions about gender

The facilitator read the riddle to the participants and showed it either on a screen or paper in order to let participants to read it as many times as they need. Once the group solved the riddle, ie. Figures out that the surgeon - a woman - is the boy's mother, facilitator leaded a discussion about the assumptions people make concerning the professional roles of women and men.

Riddle: "I cannot operate on this child"

- "Deepak and his son Arjun live in one of big cities of India. One Sunday, they take the car to go to the market. On the way, they have a serious accident and Deepak is instantly killed. His son, injured and unconscious, is rushed to the nearest hospital."[8]

Most participants said the surgeon is a mother, because the father got caught in the accident although the text does not specify this, but the name suggested it. Regarding the ethics of operating or not owning a child, she thinks that a mother cannot.

- V. believes that the surgeon "can't operate because there is intense emotional affection." "Because of the emotional shock and the likelihood that you will be mistaken for the technique, in the child's life or success will be doubtful" "(C.G).

- M.A.M says that "Mum was the surgeon and could not operate that child because he was subjectively involved. Perhaps if the father was a surgeon, he could not"

- To the question "Since a father could operate and a mother did not, a response was," the surgeon is allowed to operate the baby, but regardless of gender, whether it is mother or child's father, this I this will be much subjectively and because of feelings refused surgery "(NG), while another opinion is that "mother, because of attachment to the child, can't emotionally detach from the situation."

\subsection{Reflection on Societal Views about Women and Men's Roles}

The Aim of this activity has to help participants clarify their personal views and beliefs about the roles sixth reason point out that teacher have some. For the first activity, facilitators taped a sheet of paper marked 'agree' on one wall of the room and a sheet marked 'disagree' on the opposite wall.

Participants were said that facilitators would be reading aloud a series of statements about the role and status of women. As each statement was read, participants had to decide whether they agreed or disagreed with the statement and quickly move to the wall that indicated the opinion they favor. Those grouped together under the same sign did discuss their reasons for agreeing or disagreeing and appointed a reporter to share their reasons with the other group.

At the question "Do you think girls are better at humanity, and boys are better at science?" same answers has:

- Yes (the woman is sociable and action-oriented; I think the woman is "built" based on feelings; women are predominantly rational, and men are more attached to the goal; since primary classes, girls are interested in studying while boys on technique, skill) 
- No (I think it's about everyone's mentality and his / her ambition; everyone is different, so sex does not determine your abilities; they have the same qualities and defects; We are born with the same capabilities that the menu engages or inhibits; it does not matter sex in any field)

- There are no good men on the social side and women who are good on the logical side

- It is not mandatory. Gender is not a determining factor in terms of knowledge, skills.

- The humanitarian and scientific side is equally developed in women

Question: Why do you think men choose a STEAM profession? Answers:

- Because of their natural nature, it instinctively comes to them

- Somewhat encouraged from the outside, the media

- Yes-I think every person has his own reasons. The difference does not make it the kind

- It's not a special choice

- Because of their skills, their skills have been stimulated since childhood.

- Not only men choose them, they also do not all choose those professions.

- I do not choose men specially

- The inclinations they find

- That's what they want

- Because they are based on logic in most of the time

- Men choose these professions because they are subject to stereotypes because they are passionate and capable. This statement does not exclude the competence and passions of women.

- Because they are encouraged to do so

- They have been educated, they have to turn to the right jobs

Summing up, results show that students agree about the reasons why boys are more oriented towards STEAM. Some of them are that boys feel more comfortable with STEAM as they have been taught in STEAM skills.

2.4. Role Plays to identify Gender Stereotypes in scholastic situations

This exercise was designed to get from participants their personal views about different Gender Stereotypes treatment identified in scholastic situations.

We explained to participants that they would carry out a role play activity to help them to think about different situations in which they felt that they received different treatments due their condition of girl or boy. As participants rehearsed and discussed their scenarios, facilitator circulated and helped them get through rough patches. Then, they come together and shared their scenario with the group.

Results show that teachers treat in different way boys and girls, based (and perpetuating) on stereotypes such as that girls are more fragile and gentle (and passive) and boys more rude.

\subsection{Feedback of activity's}

Following the first Workshop, the participants expressed their views:

- "The theme was real. Gender differences exist and can be overcome by will, freedom of expression, work and education. The choices belong to us, so we have to choose wisely"

- "I hope that in the near future we will eliminate the prejudices and the stereotypes that we have today. WOMAN IS BEAUTIFUL AS A MAN! "

- "I liked the subject, I think we would have a better world if we eliminate the stereotypes, because anything a man can do can make a woman with little exceptions. I liked workshop and the way this subject was discussed and presented."

- "E-STEAM is a good initiative that I hope will help the company continue to ignore prejudices and stereotypes. Both women and men should be equal in chances to choose a career. "

- "Inequality between women and men exists on the day of sharpening. Women are devalued very much. Changing this cause could change in the future. This cause comes from grandparents' times. "(C.R.A.)

- "My opinion is that at the moment the role of the man and the role of the woman is denaturant. First, taking into account the natural role presented in the Bible, both men and women have predominant, special qualities, which distinguish them. Secondly, it is obvious that from a physical point of view a woman cannot exceed a man's strength. Equally, at the psychic level, it is demonstrated that 
the woman is focused on details and the man on the generalities, in case a problem occurs, need to be solved. Last but not least, within a family, a woman naturally fulfills her household chores easily, while a barbarian is difficult to cope with each of them. Returning to the special qualities, the woman is sensitive, tender, patient and imam, while the man presents strength, strength, self-confidence, and ease of doing business. (B.V.A.)

- "I think that there is equality between a man and a woman, in general, that if we enter into details things could degenerate from one person to another, and the conception of each one is related to several factors starting from the most elementary, the family and here many others - religion, conception, society, etc. No one stop a woman from pursuing a career that is considered to be "destined" to men, and if she wants, she is absolutely achievable. From a legal point of view, we are all equal, the rest are only perceptions and indoctrinated ideas. "

- "In conclusion, inequality between sexes does not exist in the law, at least in us. Inequality is something about your own conduct and mentality."

- "I think this subject is very varied and it takes more time to debate it."

\section{CONCLUSIONS}

The stereotypes identified during the workshop, regarding "personality" attributes are: girls are more focused on appearance, are fragile, too emotive since boys are more logical and strong. Regarding way of behaving participants says that girls are inferior to boys in sports, are neater and better organized, and are more passive, gentler. Regarding the way of expressing emotions, girls are more emotional, which might impede them doing their job.

Efforts have been made in recent years to promote STEAM education, but we still live with stereotypes that are part of the nation's culture and the way we were raised. STEAM education can change the thinking- students can be engaged in more activities for a better life together- Women and men's

Professional development should include methods for modeling and fostering creativity in the classroom. [2]

It's interesting the point of view of authors Ho, La, Nguyen, Pha, Vuong, Vuong, Pham,
Hoang \& Vuong:"In Confucian cultures, those who did not have to do handicrafts were usually government officials, considered to be part of the intelligence. Such people with letters would have better resources to secure their children with a scientific culture, as well as to impose strict disciplines, manners, and labels." [4]

Increased education may in some ways heighten and in other ways reduce women's vulnerability to the health effects of gender discrimination. [9] [10] [11]

\section{REFERENCES}

1. Margaret E. Madden, Marsha Baxter, Heather Beauchamp, Kimberley Bouchard, Derek Habermas, Mark Huff, Brian Ladd, Jill Pearon, Gordon Plague, Rethinking STEM Education: An Interdisciplinary STEAM Curriculum, Procedia Computer Science, Volume 20, 2013, Pages 541546, ISSN 1877-0509, https://doi.org/10.1016/j. procs.2013.09.316 accesed in february 2021 from https://www.sciencedirect.com/science/article/ pii/S1877050913011162

2. Elaine Perignat, Jen Katz-Buonincontro, STEAM in practice and research: An integrative literature review, Thinking Skills and Creativity, Volume 31, 2019, Pages 31-43, ISSN 1871-1871, https://doi. org/10.1016/j.tsc.2018.10.002. accesed in february 2021 from https://www.sciencedirect.com/ science/article/pii/S1871187118302190

3. Hau-lin Tam, Angus Yuk-fung Chan, Oscar Long-hin Lai, Gender stereotyping and STEM education: Girls' empowerment through effective ICT training in Hong Kong, Children and Youth Services Review, Volume 119, 2020, 105624, ISSN 0190-7409, https://doi.org/10.1016/j. childyouth.2020.105624. Accessed in 28.02.2021 from https://www.sciencedirect.com/science/ article/pii/S0190740920320478.

4. Manh-Toan Ho, Viet-Phuong La, Minh-Hoang Nguyen, Thanh-Hang Pham, Thu-Trang Vuong, Ha-My Vuong, Hung-Hiep Pham, Anh-Duc Hoang, Quan-Hoang Vuong, An analytical view on STEM education and outcomes: Examples of the social gap and gender disparity in Vietnam, Children and Youth Services Review, Volume 119, 2020, 105650, ISSN 0190-7409, https://doi.org/10.1016/j. childyouth.2020.105650. Accessed on February 2021 from https://www.sciencedirect.com/ science/article/pii/S0190740920320739

5. Lara Perez-Felkner, John S. Felkner, Samantha Nix,Melissa Magalhães, Thepuzzling relationship 
between international development and gender equity: The case of STEM postsecondary education in Cambodia, International Journal of Educational Development, Volume 72, 2020, 102102, ISSN 0738-0593, https://doi.org/10.1016/j. ijedudev.2019.102102. Accessed at 28.02.2021 from https://www.sciencedirect.com/science/ article/pii/S0738059319300756

6. Gheorghita M. Faitar, Silviu L. Faitar, Gender Gap and Stem Career Choices in 21st Century American Education, Procedia - Social and Behavioral Sciences, Volume 106, 2013, Pages 1265-1270, ISSN 1877-0428, https://doi.org/10.1016/j. sbspro.2013.12.142. Accesed in 27022021 from https://www.sciencedirect.com/science/article/ pii/S1877042813047654

7. Judith M. Delaney, Paul J. Devereux, Understanding gender differences in STEM: Evidence from college applications, Economics of Education Review, Volume 72, 2019, Pages 219238, ISSN 0272-7757, https://doi.org/10.1016/j. econedurev.2019.06.002 accessed in february 2021 from https://www.sciencedirect.com/ science/article/pii/S0272775719301761
8. Gender Bias Exercise can be accesed at https:// www.coursehero.com/file/21592978/GenderBias-Exercise-1/

9. Matthew A. Andersson, Catherine E. Harnois, Higher exposure, lower vulnerability? The curious case of education, gender discrimination, and Women's health, Social Science \& Medicine, Volume 246, 2020, 112780, ISSN 0277-9536, https://doi. org/10.1016/j.socscimed.2019.112780. Accesed in 27.02.2021 from https://www.sciencedirect.com/ science/article/pii/S0277953619307750

10. Delcea C., Siserman C., 2020: Validation and Standardization of the Questionnaire for Evaluation of Paraphilic Disorders. Rom J Leg Med28(1)14-20(2020) DOI:10.4323/rjlm.2020.14. Romanian Society of Legal Medicine.

11. Delcea C., Muller-Fabian A., Radu C. C., PerjuDumbravă D., 2019: Juvenile delinquency within the forensic context. Rom J Leg Med [27] 366-372 [2019] DOI: 10.4323/rjlm.2019.366. 2019 Romanian Society of Legal Medicine. 